\title{
Diagnostic accuracy of a urine lipoarabinomannan strip-test for TB detection in HIV-infected hospitalised
}

\section{patients}

\author{
Jonathan G. Peter*, Grant Theron*, Richard van Zyl-Smit*, \\ Asheen Haripersad*, Lynelle Mottay*, Sarah Kraus*, Anke Binder*, \\ Richard Meldau*, Anneli Hardy ${ }^{\#}$ and Keertan Dheda*, ${ }^{\star,+}$
}

ABSTRACT: Lack of point-of-care tests for tuberculosis (TB) result in diagnostic delay, and increased mortality and healthcare-related costs.

The urine Determine ${ }^{\text {TM }}$ TB-LAM point-of-care strip-test was evaluated in 335 prospectivelyrecruited hospitalised patients with suspected TB-HIV co-infection (group 1) and from 88 HIVinfected hospitalised patients with non-TB diagnoses (group 2). Cut-off point-specific analyses were performed using: 1) a microbiological reference standard (culture positive versus negative); and 2) a composite reference standard (exclusion of patients with clinical-TB from the culturenegative group).

Using the microbiological reference and the manufacturer-recommended grade-1 cut-off point, LAM sensitivity and specificity was $66 \%$ (95\% Cl 57-74\%). By contrast, using the composite reference sensitivity was $60 \%(95 \% \mathrm{Cl} 53-67 \%)$ and specificity improved to $96 \%(95 \% \mathrm{Cl} 89-100 \%)$ $(p=0.001)$. The same pattern was seen when the grade-2 cut-off point was used (specificity $75 \%$ versus $96 \% ; p=0.01$ ). In group two patients specificity was poor using the grade-1 cut-off point, but improved significantly when the grade-2 cut-off point was used ( $90 \%$ versus $99 \% ; p=0.009$ ). The grade-2 cut-off point also offered superior inter-reader reliability $(p=0.002)$. Sensitivity was highest in those with a CD4 $<200$ cells per $\mathrm{mL}$. LAM combined with smear-microscopy was able to rule-in TB in $71 \%$ of Mycobacterium tuberculosis culture-positive patients.

This preliminary study indicates that the LAM strip-test may be a potentially useful rapid rule-in test for TB in hospitalised patients with advanced immunosuppression. The grade 2, but not the manufacturer-recommended grade 1 cut-off point, offered superior rule-in utility and inter-reader reliability. Larger studies to evaluate cut-off points and diagnostic accuracy are urgently required.

KEYWORDS: Diagnostic, lateral flow assay, lipoarabinomannan, point-of-care

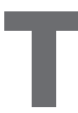

he co-epidemics of tuberculosis (TB) and HIV remain out of control in sub-Saharan Africa [1]. TB is still the leading cause of HIV-related mortality [2] and up to $80 \%$ of newly diagnosed TB cases in sub-Saharan Africa are HIV co-infected [1]. African hospitals are overwhelmed by admissions of TB-HIV co-infected patients, hospital stay is prolonged because of suboptimal diagnostic tools and, consequently, healthcarerelated costs are high [3]. A recent South African study showed that the early commencement of anti-TB treatment decreased 8-week mortality and length of stay in hospitalised TB patients [4].
An autopsy study of 240 in-patients (HIV seroprevalence rate of $94 \%$ ) found that, of those not on anti-TB treatment, $>40 \%$ were culture positive for Mycobacterium tuberculosis at the time of death [5]. Thus, delay or failure to diagnose TB is associated with high mortality in HIV co-infected hospitalised patients. Traditional TB diagnostic tools perform poorly in these patients $[6,7]$, and novel molecular diagnostics such as Xpert MTB/RIF (Cepheid, Sunnyvale, CA, USA) and other diagnostic adjuncts such as bronchoscopy, remain unaffordable and/or limited by the need for specialised laboratories and training [8]. Moreover, current tools perform
AFFILIATIONS

*Lung Infection and Immunity Unit, Division of Pulmonology \& UCT Lung Institute, Dept of Medicine, University of Cape Town,

\#Dept of Statistical Sciences, University of Cape Town, and -Institute of Infectious Diseases and Molecular Medicine, University of Cape Town, Cape Town, South Africa. +Dept of Infection, University College London Medical School, London, UK

CORRESPONDENCE

K. Dheda

University of Cape Town

H47 Old Main Building

Groote Schuur Hospital

Observatory

7925 Cape Town

Cape Town

South Africa

E-mail: Keertan.Dheda@uct.ac.za

Received:

Nov 182011

Accepted after revision:

Feb 082012

First published online:

Feb 232012

European Respiratory Journal Print ISSN 0903-1936

Online ISSN 1399-3003 
poorly in sputum-scarce and extrapulmonary (EP) TB. There remains a pressing need for a rapid, inexpensive and simple point-of-care (POC) TB diagnostic for HIV co-infected patients.

Lipoarabinomannan (LAM) is a $17.5 \mathrm{kDa}$ glycolipid that forms a component of the outer cell wall of mycobacterial species [9]. It is filtered by the kidney and detectable in the urine, and has been extensively evaluated as a TB diagnostic antigen [10-15]. A urine ELISA-based kit to detect LAM antigen (Clearview ${ }^{\circledR}$ TB-ELISA; Alere, Waltham, MA, USA) was found to be clinically useful in hospitalised TB-HIV co-infected patients with advanced immunosuppression [16], and was shown to be more sensitive than smearmicroscopy in a TB screening study prior to the introduction of highly active antiretroviral therapy (HAART) [17].

The ELISA version of the test has now evolved into a simple POC urine immunochromatographic strip-test (lateral flow assay). The commercial version of the strip-test, which was evaluated in this study, is planned for release in the first quarter of 2013. This urine LAM strip-test is marketed by Alere, USA as the Determine ${ }^{\circledR}$ TBLAM Ag rapid test (fig. 1). This novel test requires only $60 \mu \mathrm{L}$ of unprocessed urine, can be performed at the bedside, requires little technical skill, no power source and provides a result within $25 \mathrm{~min}$ at a probable cost of less than US\$3.50 (the locked-in final version evaluated here is to be launched in quarter 1 of 2013) [18]. A recent evaluation of the LAM strip-test in outpatients being screened for antiretroviral therapy at primary care clinics showed promising results, especially for patients with advanced immunosuppression [19]. However, performance amongst hospitalised HIV-infected patients with advanced immunosuppression, in whom rapid diagnosis and treatment could potentially impact mortality remains unknown. Thus, we undertook a study to

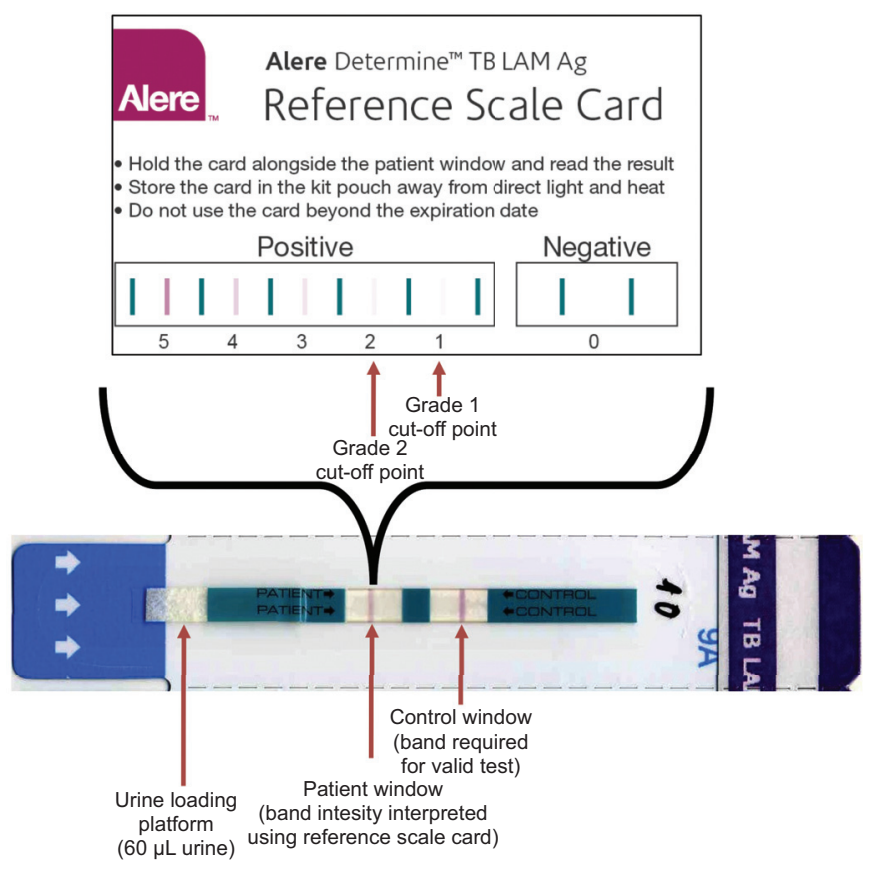

FIGURE 1. Lipoarabinomannan (LAM) strip-test and reference scale card. The reference scale card (courtesy of Alere, Waltham, MA, USA) illustrates six cut-off points (visual grades $0-5$ ) categorised by different band intensities appearing in the patient window. prospectively evaluate the diagnostic accuracy of the LAM striptest in hospitalised HIV co-infected patients with suspected TB. Some of the preliminary results of these studies, using the manufacturer-recommended cut-off point, have been previously reported in the form of an abstract [16].

\section{METHODS}

\section{Study population}

The study population, divided into two groups, consisted of 423 adult patients recruited from four hospitals (three districtand one tertiary-level hospital; Groote Schuur, GF Jooste, Victoria and Somerset Hospitals, Cape Town, South Africa) between July 22, 2009 and December 14, 2010 in Cape Town, South Africa. Study group 1 consisted of 335 prospectively recruited hospitalised individuals with suspected TB and HIV, while study group 2 consisted of $88 \mathrm{HIV}$-infected hospitalised non-TB patients (possessing alternative diagnoses and no clinical or microbiological evidence of TB). Group 2 patients were recruited to further evaluate test specificity due to the limited number of confirmed non-TB patients in group 1. A study and analysis outline is shown in figure 2. Group 1 hospitalised patients were consecutively recruited following referral by clinicians who considered patients to be HIVinfected and with suspected TB. Group 2 patients represented a convenience sample and were specifically recruited as they were not suspected of having TB. Only three patients, who refused consent, were excluded from the study. All patients provided informed consent and the study was approved by the University of Cape Town Faculty of Health Sciences Human Research Ethics Committee. Clinical information documented for enrolled patients included demographic information, past history of TB, symptoms and vital signs, HIV status, renal function, and details about anti-TB therapy.

The clinical management of all patients (including the timing and extent of diagnostic work-up, decision to commence anti-TB treatment and final discharge from hospital) was undertaken by consultant-led groups of health care workers who had no association with the study team. TB diagnostic work-up was not standardised and was at the discretion of the attending senior physician. Table 1 provides a site-specific breakdown of clinical samples collected for TB diagnosis. The local reference laboratory processed all clinical specimens collected for routine TB diagnosis (fluorescence smear microscopy on NALC $/ \mathrm{NaOH}$ processed and concentrated samples followed by liquid culture (BACTEC MGIT 960; BD Diagnostics, Franklin Lakes, NJ, USA) were performed when appropriate).

\section{LAM methodology}

All patients were required to give a spot urine sample $(10-30 \mathrm{~mL})$ collected in a sterile container as soon as possible after recruitment. A urine dipstick test (UriCHECK 9; RapiMed Diagnostics, Pretoria, South Africa) was immediately performed to assess for protein, blood and leukocytes. Urine was stored at $-20^{\circ} \mathrm{C}$ for later batched testing. The LAM strip-test (a single manufacturing lot \#101102) was performed on unprocessed thawed urine according to manufacturer's instructions and after 25-35 min, two readers, blinded to clinical details and TB status, independently evaluated the LAM strips for all study patients. The detailed methods of LAM strip-test grading using the manufacturer's reference card (fig. 1), the interpretation of 


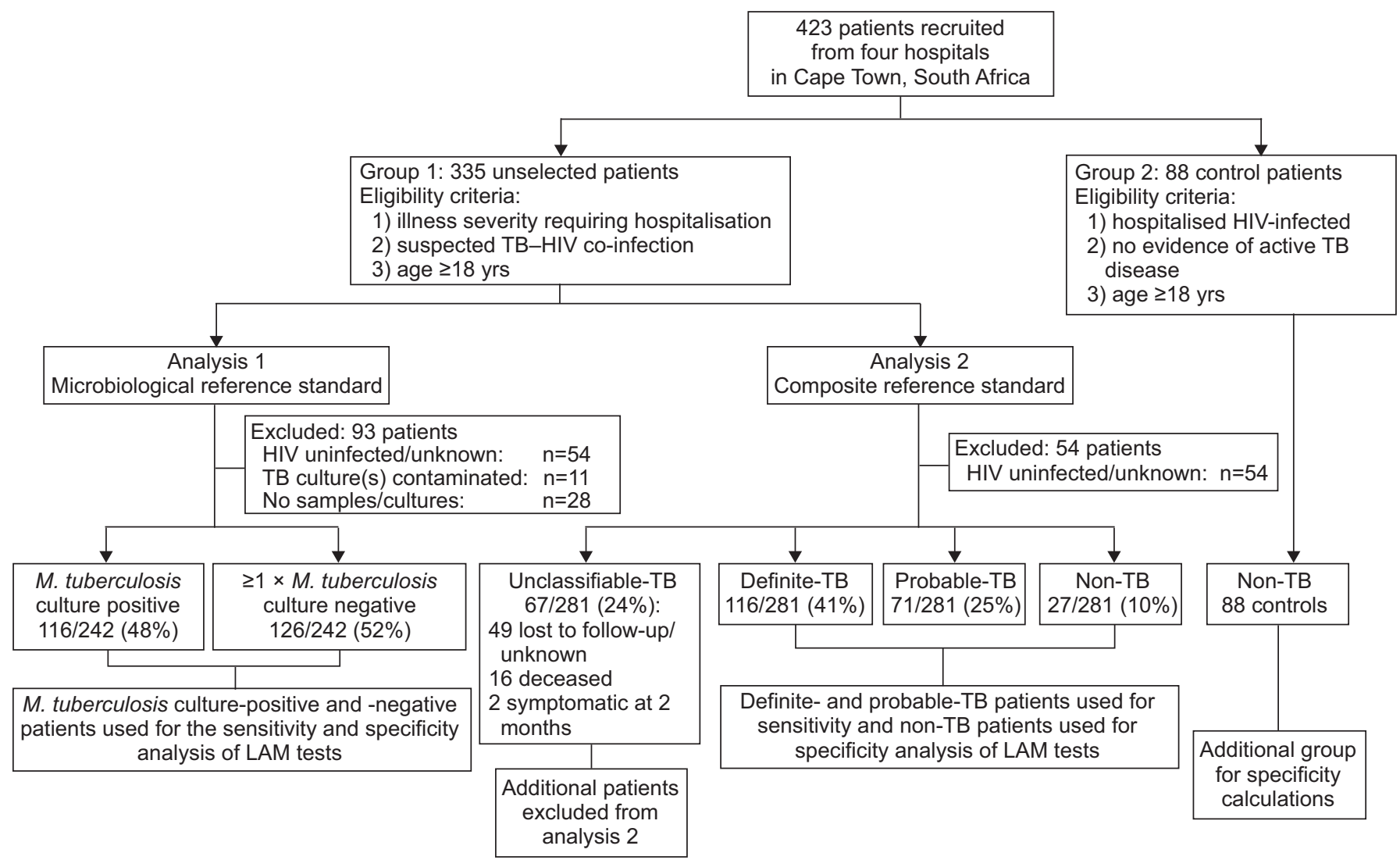

FIGURE 2. Study population and outline of different patient groups used for the two analyses of urine lipoarabinomannan (LAM) strip-test diagnostic accuracy. M. tuberculosis: Mycobacterium tuberculosis; TB: tuberculosis.

varying test results, and the further experiments performed to better evaluate inter-reader reliability are provided in the online supplementary material. Concurrently, the Clearview TB ELISA was performed on all samples, as previously described, by a technician blinded to patient TB diagnostic status [20].

\section{Definition of the reference standards and analytical approach}

Given the limitations of liquid TB culture in hospitalised HIVinfected patients with advanced immunosuppression (low sputum baciliary load, sputum-scarce disease and EPTB) a composite reference standard was used to categorise patients. Patients were categorised into the following diagnostic groups based on a combination of smear and culture results, clinical treatment and 2-month follow-up, and radiology findings.

\section{Definite-TB}

At least one $M$. tuberculosis sample positive by liquid culture (either sputum or non-sputum). Definite-TB cases were subclassified by whether only sputum, non-sputum or both sputum and non-sputum samples were TB culture positive into pulmonary TB (PTB), EPTB or combined PTB/EPTB, respectively.

\section{Probable-TB}

Not meeting the criterion for definite-TB, but with a clinicalradiological picture highly suggestive of $\mathrm{TB}$ and showing a response to anti-TB treatment at 2-month follow-up.
Non-TB

No microbiological evidence of $M$. tuberculosis and an alternative diagnosis available. Patients were not treated for TB. Patients culture positive for nontuberculosis mycobacteria and not receiving anti-TB treatment were assigned to this group.

\section{Unclassifiable-TB}

Unable to assign the patient to any of the above-mentioned diagnostic groups due to death of unknown cause (without autopsy), on-going symptoms at follow-up or loss-to-follow-up at 2 months.

Two analyses of diagnostic accuracy were performed using: 1) analysis 1 used a microbiological reference standard (culture positive versus negative); and 2) analysis 2 used a composite reference standard (probable and unclassified patients were excluded from the culture negative group). Thus, analysis 2 combined the definite-TB $(n=116)$ and probable-TB $(n=71)$ patients for the sensitivity calculation and used non-TB patients for the specificity calculation. Specificity calculations for group 2 $(\mathrm{n}=88)$ non-TB patients are presented separately. Figure 2 outlines these groups used in the two analyses.

\section{Statistical analysis}

LAM strip-test performance was evaluated using the manufacturer-provided reference card and receiver-operating characteristic (ROC) curve analysis was used to determine the optimal "rule-in" cut-off point. Test sensitivity and specificity 


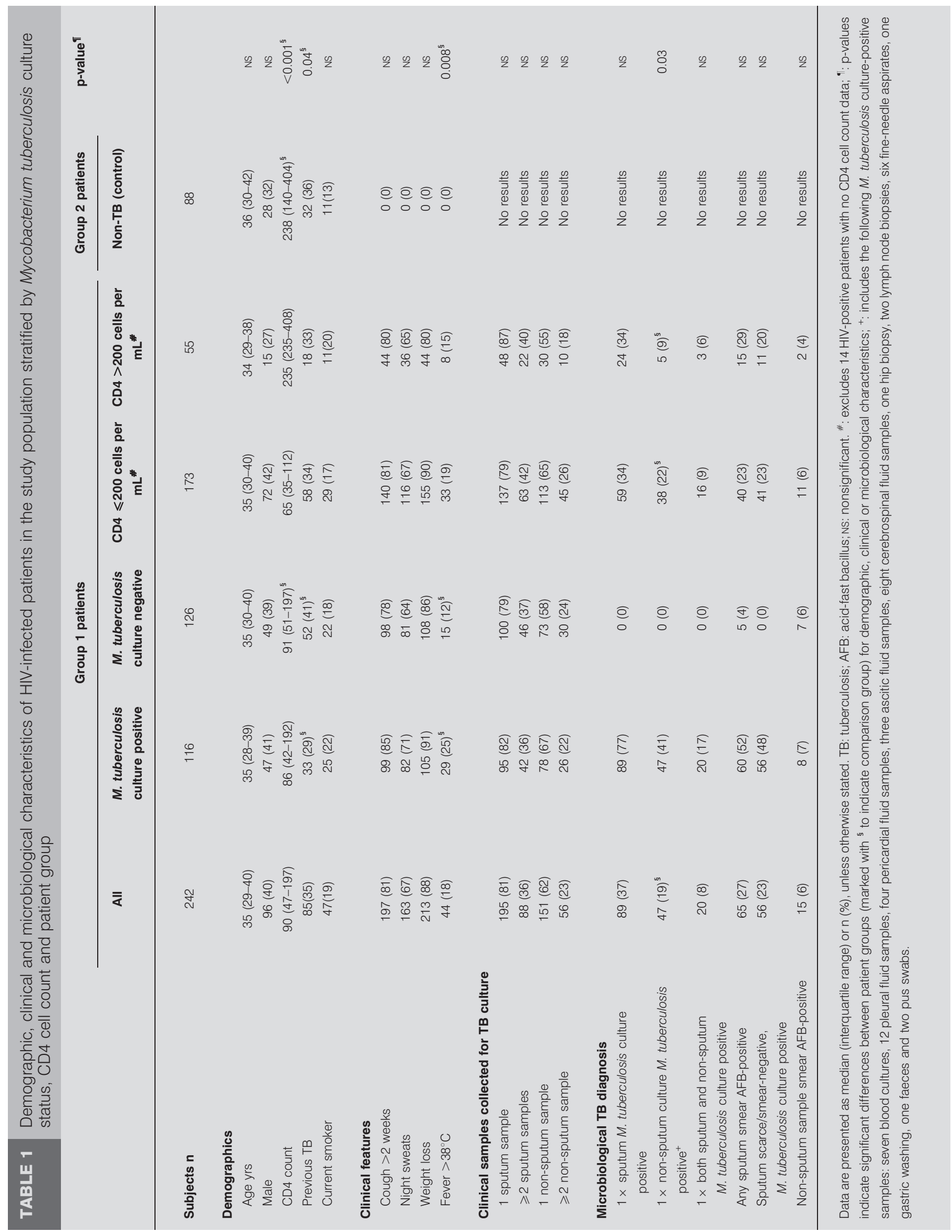


TABLE 2 Performance outcomes of smear microscopy, lipoarabinomannan (LAM) ELISA and LAM strip-test (using grade 1 and 2 cut-off points) in HIV-infected patients

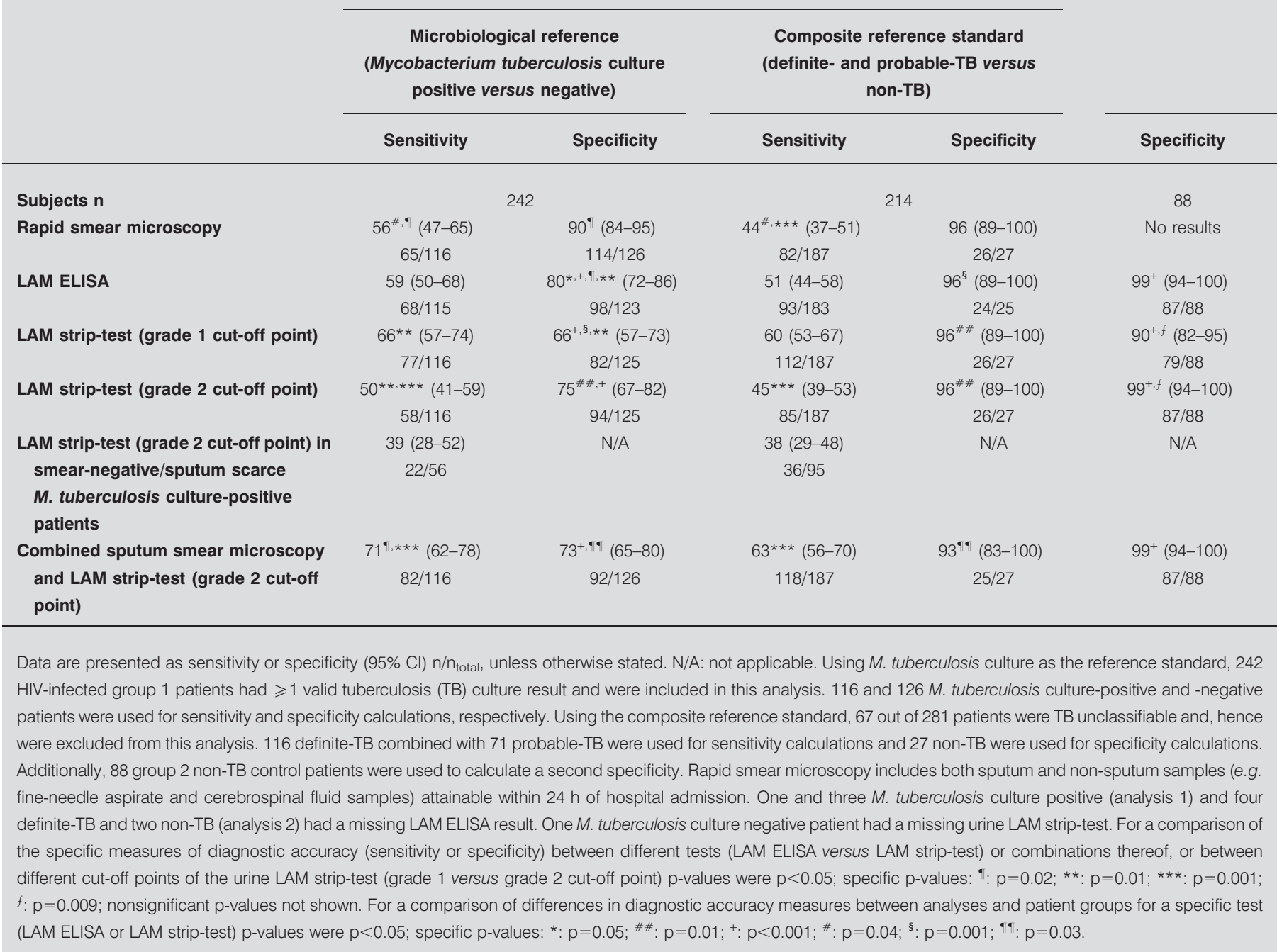

were calculated with 95\% confidence intervals (positive likelihood ratios are presented in the online supplementary results). Intra- and interobserver agreement was assessed using the Kappa statistic, and categorised as poor (0-0.20), fair (0.21-0.40), moderate $(0.41-0.60)$, good $(0.61-0.80)$ and very good $(0.81-1)$ [21]. Additionally, a McNemar Chi-squared test was used to compare the difference in agreement between readers at the grade 1 and 2 cut-off points. STATA IC, version 10 (StataCorp, College Station, TX, USA) was used for all statistical analyses. Study reporting and analysis were consistent with the Standards for the Reporting of Diagnostic Accuracy (STARD) criteria [22].

\section{RESULTS}

Demographic, clinical and microbiological characteristics of study patients (groups 1 and 2)

HIV infection was confirmed in 281 (84\%) out of 335 of group 1 patients and consequently, $54 \mathrm{HIV}$-uninfected group 1 patients were excluded from the analyses. The demographic, clinical, diagnostic work-up and microbiological details of the HIV-infected patients stratified by culture status, patient group and CD4 cell count are shown in table 1. The median (interquartile) CD4 cell count in group $1 \mathrm{M}$. tuberculosis culture-negative HIV-infected patients was significantly lower than the group 2 non-TB patients (91 (51-197) versus 238 (140-404) cells per $\mathrm{mL} ; \mathrm{p}<0.001)$. Of all clinical symptoms and signs, only an admission fever $>38^{\circ} \mathrm{C}$ was associated with M. tuberculosis culture positivity $(\mathrm{p}=0.008)$.

$116(48 \%)$ out of 242 of group $1 \mathrm{HIV}$-infected patients were $M$. tuberculosis culture positive. Using the composite reference, a further 71 patients were categorised as probable-TB (nine sputum/non-sputum sample smear positive, $M$. tuberculosis culture negative/contaminated). Given suspected TB-HIV coinfection, attending clinicians attempted, where possible, to collect both sputum and non-sputum samples for TB diagnosis, with 195 (81\%) out of 242 and 151 (62\%) out of 242 of all group-1 HIV-infected patients having at least one sputum and nonsputum sample. Of the HIV-infected $M$. tuberculosis culturepositive patients, $69(59 \%)$ out of 116 were sputum culture 


\begin{tabular}{|c|c|c|c|}
\hline \multirow[t]{2}{*}{ Diagnostic test(s) } & \multicolumn{3}{|c|}{ M. tuberculosis culture-positive group 1 patients } \\
\hline & PTB only & PTB and EPTB & EPTB only \\
\hline Subjects $n$ & 69 & 20 & 27 \\
\hline LAM strip-test (grade 1 cut-off point) & $67(55-77) 46 / 69$ & $80(58-92) 16 / 20$ & $59(41-76) 16 / 27$ \\
\hline LAM strip-test (grade 2 cut-off point) & 51 (39-62) 35/69 & 60 (39-78) 12/20 & $41^{\# \#}(25-59) 11 / 27$ \\
\hline $\begin{array}{l}\text { LAM strip-test (grade } 2 \text { cut-off point) in smear-negative/ } \\
\text { sputum scarce } M \text {. tuberculosis culture-positive patients }\end{array}$ & $35(19-55) 8 / 23$ & $50(19-81) 3 / 6$ & $32(16-53) 7 / 22$ \\
\hline $\begin{array}{l}\text { Combined sputum smear microscopy and LAM strip-test } \\
\text { (grade } 2 \text { cut-off point) }\end{array}$ & 78 (67-86) 54/69 & $85(64-95) 17 / 20$ & $44^{f}(28-63) 12 / 27$ \\
\hline
\end{tabular}

Data are presented as sensitivity $(95 \% \mathrm{Cl}) \mathrm{n} / \mathrm{n}_{\text {total }}$, unless otherwise stated. PTB: pulmonary tuberculosis (TB); EPTB: extrapulmonary TB. ${ }^{\#:} 116$ definite-TB patients were used for calculations (69 patients with at least one culture-positive sputum, 20 patients with at least one culture-positive sample from sputum and another non-sputum site and 27 culture positive from a non-pulmonary site, i.e. not from sputum). ": Rapid (within $24 \mathrm{~h}$ of hospitalisation) smear microscopy includes both sputum and nonsputum samples (e.g. fine-needle aspirate and cerebrospinal fluid samples). ${ }^{+}$: one M. tuberculosis culture-positive patient was missing a LAM ELISA result. For a comparison of test sensitivity in specific TB disease states between different tests (LAM ELISA versus LAM strip-test) or combinations thereof, or between different cut-off points of the urine LAM strip-test (grade 1 versus grade 2 cut-off point) $p$-values were ${ }^{\S}: p=0.01 ;^{f}: p=0.04 ;{ }^{\# \#}: p=0.07$ (if analysis restricted to sputum smear microscopy only then $p<0.001$ ). ${ }^{* *}: p<0.001$ for a comparison of a specific test (LAM ELISA or LAM strip-test) in different TB disease states.

positive only (PTB), $27(23 \%)$ out of 116 were non-sputum culture positive only (EPTB) and $20(18 \%)$ out of 116 were culture positive on both sputum and non-sputum samples (PTB/EPTB). Overall, 56 (48\%) out of 116 of M. tuberculosis culture-positive patients were either sputum scarce or sputum smear-negative (21 (38\%) out of 56 sputum scarce and $35(62 \%)$ out of 56 sputum smear-negative).

The performance outcomes for rapid smear microscopy stratified by patient group, analysis 1 and 2, TB diagnostic subtype and CD4 cell count are shown in tables $2-4$. Using the microbiological reference (analysis 1), the sensitivity (95\% CI) of rapid smear microscopy was 56\% (47-65\%), while using the composite reference (analysis 2) sensitivity (95\% CI) was $44 \%$ $(37-51 \%)(p=0.04)$. The sensitivity $(95 \% \mathrm{CI})$ of rapid smear microscopy was significantly lower in EPTB compared with PTB $(19 \%(8-37 \%)$ versus $67 \%$ (55-77\%), $\mathrm{p}<0.001)$ (table 3$)$. Given the low diagnostic yield of smear microscopy, 118 (49\%) out of 242 of unselected HIV-infected group 1 patients underwent specialised radiology to aid diagnostic decision-making (92 had abdominal ultrasound, 12 had computed tomography of the brain and 27 had echocardiography with/without pericardiocentesis).

\section{Performance of the LAM strip-test using different cut-off points}

Reader-specific performance outcomes and ROC analysis for both readers for analysis 1 and 2 are displayed in figure 3 . In the analysis 1 (fig. 3a), using the manufacturer-suggested grade 1 cut-off point, the sensitivity, specificity and positive likelihood ratio (LR+) for reader 1 was $63.8 \%, 72.8 \%$ and 2.3 , respectively, and for reader 2 was $66.4 \%, 65.6 \%$ and 1.9 , respectively. Using a grade 2 or 3 cut-off point to optimise test rule-in value results in a reduced test sensitivity at both cut-off points (reader 1: 74 $(63.8 \%)$ out of 116 versus 53 (45.7\%) out of 116 (grade 2 ) and 47 ( $40.5 \%$ ) out of 116 (grade 3 ), $p=0.005$ and $p<0.001$, respectively) and an increased test specificity (reader 1: 91 (72.8\%) out of 125 versus 101 (80.8\%) out of 125 (grade 2) and 109 (87.2\%) out of 125 (grade 3), $\mathrm{p}=0.13$ and $\mathrm{p}=0.004$, respectively). By contrast, in analysis 2 (fig. 3b), using the manufacturer-suggested grade 1 cut-off point, the sensitivity, specificity and LR+ for reader 1 was $56.7 \%, 92.2 \%$ and 7.2 , respectively, and for reader 2 was $59.9 \%$, $91.3 \%$ and 6.9 , respectively. Using a grade 2 cut-off point to optimise rule-in utility, improved test specificity for both readers (reader 1: 106 (92.2\%) out of 115 versus 113 (98.3\%) out of $115, p=0.03$; reader 2 : $105(91.3 \%)$ out of 115 versus $113(98.3 \%)$ out of 115, $\mathrm{p}=0.02$ ) and consequently increased LR+.

\section{Performance outcomes of the LAM strip-test with and without smear microscopy}

Tables 2-4 shows diagnostic accuracy measures for LAM ELISA and strip-test in all HIV-infected patients with results presented for both analysis 1 and 2, and with group 1 and 2 patients separately, and results stratified by TB diagnostic subtype or CD4 cell count. Additional tables in the online supplementary results section present data from analysis 1 and 2 separately (including LR+ values).

\section{Analysis 1}

Rapid smear microscopy had similar sensitivity to the LAM strip-test (grade 2 cut-off point) $(\mathrm{p}>0.05)$. LAM strip-testing (grade 2 cut-off point) was less sensitive than smear microscopy in PTB and in the combined PTB/EPTB group, but more sensitive in EPTB alone, although the numbers did not reach significance (EPTB rapid smear: 19\% (8-37\%) versus LAM strip: $41 \%(25-59 \%), p=0.07)$. However, if smear microscopy in the EPTB group was restricted to sputum smear microscopy only, then LAM strip-testing offered significantly improved sensitivity compared with smear microscopy: 41\% (25-59\%) (11 out of 27 ) versus $0 \%$ ( 0 out of 27$), p<0.001$. The sensitivity of the 


\begin{tabular}{|c|c|c|c|c|c|c|c|c|c|}
\hline 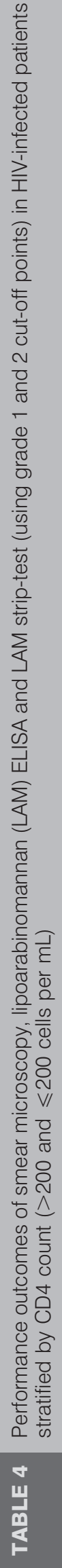 & 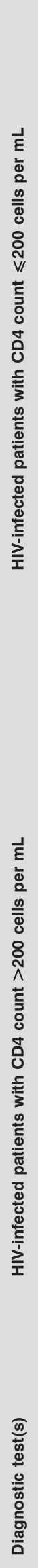 & 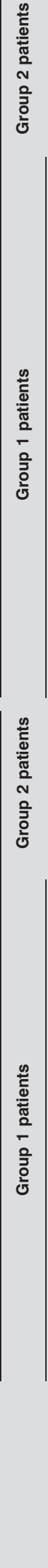 & 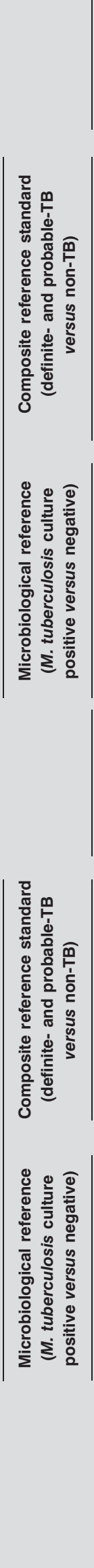 & 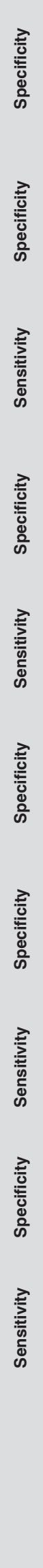 & 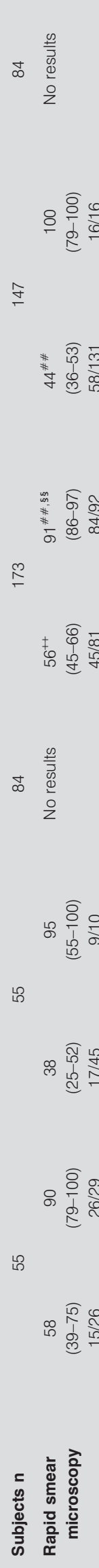 & 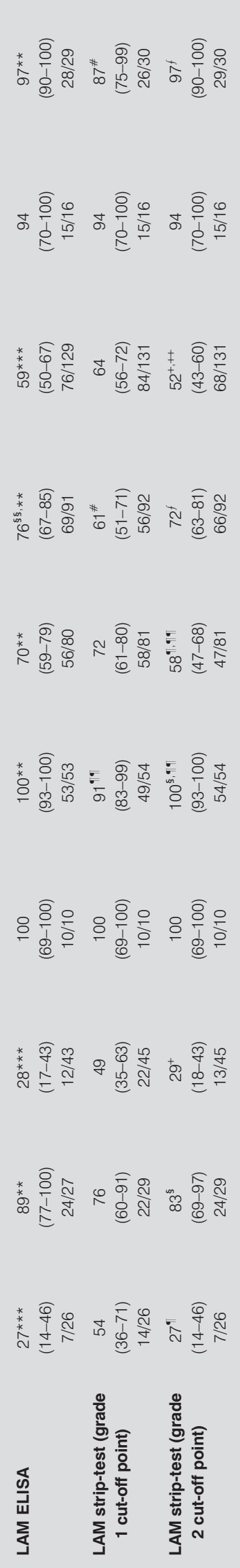 & 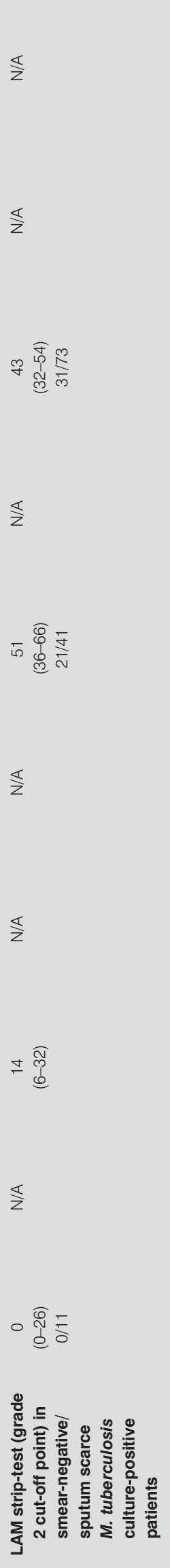 & 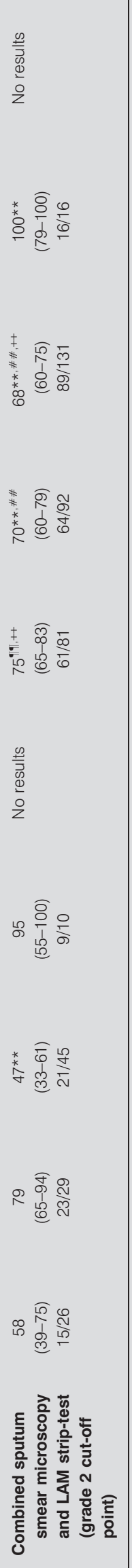 & 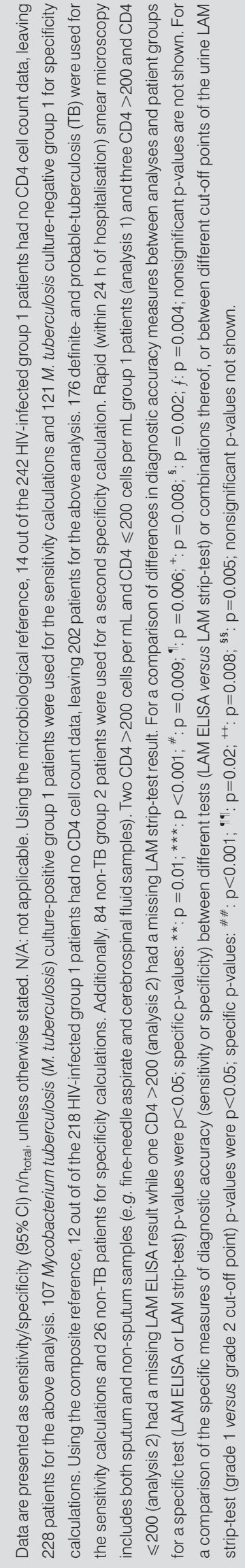 \\
\hline
\end{tabular}




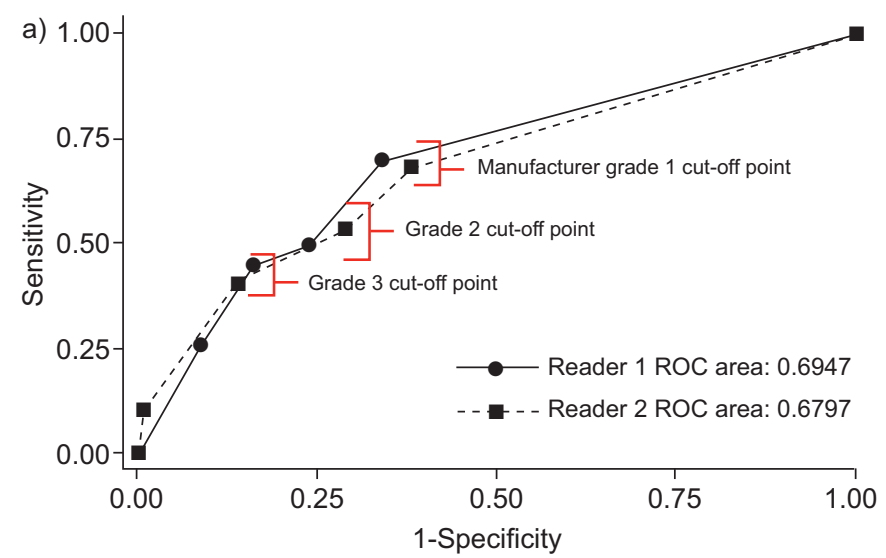

Cut-off point
Grade 1
Sensitivity
Specificity
Positive likelihood ratio
Grade 2
Sensitivity
Specificity
Positive likelihood ratio
Grade 3
Sensitivity
Specificity
Positive likelihood ratio

Reader 1

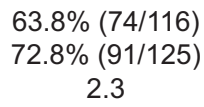
$45.7 \%(53 / 116)$
$80.8 \%(101 / 125)$ 2.4

$40.5 \%(47 / 116)$

$87.2 \%(109 / 125)$

3.2
$88 \%(110 / 125)$

3.1
$37.1 \%(43 / 116)$ b)

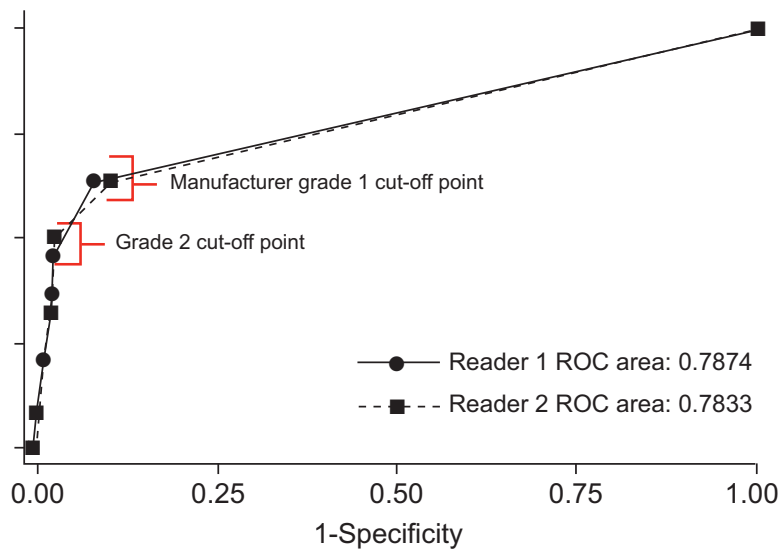

Cut-off point

Grade 1

Sensitivity

Specificity

Positive likelihood ratio

Grade 2

Sensitivity

Specificity

Positive likelihood ratio
Reader 1

$56.7 \%(106 / 187)$
$92.2 \%(106 / 115)$
7.2

$40.6 \%(76 / 187)$

$98.3 \%(113 / 115)$ 23.4
Reader 2

$59.9 \%(112 / 187)$

$91.3 \%(105 / 115)$

6.9

$45.5 \%(85 / 187)$

$98.3 \%(113 / 115)$ 26.1

FIGURE 3. Receiver operator characteristic (ROC) curves for urine lipoarabinomannan strip-test graded by two independent readers for HIV-infected patients. Sensitivity, specificity and positive likelihood ratios are shown for different visual band intensity-based cut-off points (grade 1 (manufacturer recommended) and grades 2 and 3 (optimised for "rule-in") are indicated). a) Analysis 1 (Mycobacterium tuberculosis culture reference standard). b) Analysis 2 (composite reference standard).

LAM strip-test (grade 2 cut-off point) in smear negative/sputum scarce HIV-infected TB patients was 39\% (28-52\%), improving to $51 \%(36-66 \%)$ in HIV-infected patients with a CD4 cell count $\leqslant 200$ cells per $\mathrm{mL}$, and $58 \%(41-74 \%)$ when the CD4 cell count was $\leqslant 100$ cells per $\mathrm{mL}(\mathrm{p}=0.2$ and $\mathrm{p}=0.09$, respectively; see online supplementary tables for CD4 $\leqslant 100$ cells per mL results). Overall, the sensitivity (95\% CI) of the LAM strip-test (grade 2 cut-off point) was higher in CD4 $\leqslant 200$ cells per $\mathrm{mL}$ versus CD4 $>200$ cells per $\mathrm{mL}$ ( $58 \%$ ( $47-68 \%$ ) versus $27 \%(14-46 \%), \mathrm{p}=0.006)$. No significant differences were noted for the above tests amongst patients with CD $4 \leqslant 200$ cells per $\mathrm{mL}, \leqslant 100$ cells per $\mathrm{mL}$ and $\leqslant 50$ cells per $\mathrm{mL}$ (data not shown). The combined performance of rapid smear microscopy and the urine LAM strip-test (grade 2 cut-off point) in HIV-infected patients with a CD4 cell count $\leqslant 200$ cells per $\mathrm{mL}$ was significantly better than either sputum smear microscopy or the LAM strip-test alone (75\% (65-83\%) versus $56 \%(45-66 \%)$ versus $58 \% \quad(47-68 \%)$, $\mathrm{p}=0.008$ and $\mathrm{p}=0.02)$. TB LAM ELISA results were not significantly different to LAM strip-test results at both grade 1 and 2 cut-off points. The specificity of the TB LAM ELISA and LAM strip-test (both cut-off points) in group 1 patients was $80 \%$ $(72-86 \%)$ and $75 \%(67-82 \%)$, respectively, while in group 2 patients, specificity was $99 \%$ (94-100\%) for both the LAM ELISA and strip-tests. In both CD4 $>200$ cells per $\mathrm{mL}$ and $\leqslant 200$ cells per $\mathrm{mL}$ LAM strip-test (grade 2 cut-off point), specificity was higher in group 2 than in group 1 patients (CD4 $>200$ cells per $\mathrm{mL}: 100 \%$ (93-100\%) versus 83\% (69-97\%), $\mathrm{p}=0.002$; CD4 $\leqslant 200$ cells per mL: 97\% (90-100\%) versus $72 \%(63-81 \%)$, $\mathrm{p}=0.004)$. In group 2 patients, LAM strip-test specificity was higher using the grade 2 versus grade 1 cut-off point $(100 \%$ (93-100\%) versus 91\% (83-99\%), $\mathrm{p}=0.02)$.

\section{Analysis 2}

As in analysis 1, rapid smear microscopy had similar sensitivity to the LAM strip-test (grade 2 cut-off point) ( $\mathrm{p}>0.05)$. Overall, the sensitivity of the TB LAM ELISA and LAM strip-test (both cut-off points) showed a small, but nonsignificant reduction compared with analysis 1 , but improvements between patient subgroups were similar between analyses. The sensitivity of the LAM strip-test (grade 2 cut-off point) in smear negative/ sputum scarce HIV-infected TB patients was 38\% (29-48\%), improving to $43 \%$ (32-54\%) in HIV-infected patients with a CD4 cell count $\leqslant 200$ cells per $\mathrm{mL}$, and $49 \%$ (35-63\%) when the CD4 cell count was $\leqslant 100$ cells per $\mathrm{mL}(\mathrm{p}=0.5$ and $\mathrm{p}=0.2$, respectively; see online supplementary tables for CD $4 \leqslant 100$ cells per $\mathrm{mL}$ results). The sensitivity $(95 \% \mathrm{CI})$ of the LAM strip-test (grade 2 cut-off point) was higher in CD4 $\leqslant 200$ cells per $\mathrm{mL}$ versus CD4 $>200$ cells per $\mathrm{mL}$ (52\% (43-60\%) versus $29 \%$ (18$43 \%), p=0.008)$. The combined performance of rapid smear microscopy and the urine LAM strip-test (grade 2 cut-off point) in HIV-infected patients with a CD4 cell count $\leqslant 200$ cells per $\mathrm{mL}$, was significantly better than either sputum smear microscopy or the LAM strip-test alone (68\% (60-75\%) versus $44 \%$ (36$53 \%$ ) versus $52 \%(43-60 \%), p<0.001$ and $p=0.008)$. Notably, the overall specificity of both the TB LAM ELISA and LAM striptest (both cut-off points) was higher in analysis 2 than in analysis 1 (LAM strip-test (grade 2 cut-off point) specificity: analysis 2: group 1 96\% (89-100\%) and group 2 99\% (94-100\%) versus 
analysis $1: 75 \%(67-82 \%), p=0.01$ and $p<0.001$, respectively). A similar difference in test specificities between analysis 1 and 2 was found in patients with CD4 $>200$ cells per $\mathrm{mL}$ and $\leqslant 200$ cells per $\mathrm{mL}$ (LAM strip-test (grade 2 cut-off point) specificity in CD4 $\leqslant 200$ cells per $\mathrm{mL}$ : analysis 2: group $194 \%$ (70-100\%) and group 2 97\% (90-100\%) versus analysis 1: $72 \%$ $(63-81 \%), p=0.06$ and $\mathrm{p}=0.004$, respectively).

\section{Factors associated with a positive LAM strip-test result}

A positive LAM strip-test was associated with a CD4 cell count $\leqslant 200$ cells per $\mathrm{mL}(\mathrm{p}<0.001$ for both reader 1 and 2$)$, but not with any other clinical parameter including urinalysis results, renal function results (urea and creatinine levels) and/or calculated glomerular filtration rate (using the modified Cochrane-Gault method).

\section{LAM strip-test inter- and intraobserver variability}

At the grade 1 cut-off point, the inter- and intraobserver agreement between readers was fair to good $(\kappa=0.45-0.59$ for interobserver agreement and 0.56-0.73 for intraobserver agreement). By contrast, at the grade 2 cut-off point, the inter- and intraobserver agreement improved and was moderate to very good $(\kappa=0.78-0.92$ for interobserver agreement and between $\kappa=0.92-0.96$ for intraobserver agreement). Interobserver agreement improved significantly by using a grade- 2 versus a grade- 1 cut-off point $(p=0.002)$. Detailed tables of the agreement between all the readers for different LAM strip-test grades is provided in the online supplement results section.

\section{LAM strip-test indeterminate tests}

423 LAM strip-tests were performed and read by two independent readers. The initial indeterminate rate (one test) for the LAM strip-test (defined as a broken band in the patient window) was 29-60 (7-14\%) out of 423 depending on the reader. In $>90 \%$ of these cases, a repeat LAM strip-test produced a valid result. Thus, only $1-2 \%$ of tests remained indeterminate after repeat testing. There was no significant difference if performance outcomes were calculated excluding indeterminate test results or considering them as "negative" test results. Given the proposed use of the LAM strip-test as a rapid rule-in test, the analyses presented above consider indeterminate test results as test "negative". Three failed tests occurred (no band visible in the control window) and they were successfully repeated.

\section{DISCUSSION}

In Africa, HIV-TB co-infected patients form the bulk of medical admissions, have prolonged hospital stay due to diagnostic delay, have a high mortality if treatment is not rapidly initiated and overburden public health facilities. To address these issues, we evaluated the accuracy and clinical utility of a urine POC LAM strip-test to rule-in TB in HIV-infected hospitalised adults in a resource-poor high HIV-prevalence setting. The key findings of our study were that in hospitalised HIV co-infected patients: 1) the LAM strip-test had inadequate specificity at the manufacturer's recommended cut-off point; 2) using the grade 2 cut-off point, LAM offers better reliability (minimal interobserver variability) and utility as a rule-in test; 3) LAM strip-testing offered incremental value over smear microscopy in EPTB and thus, if used in combination with smear microscopy for HIVinfected hospitalised patients, nearly three-quarters of definiteTB cases were identifiable; and 4) the LAM strip-test was positive in approximately one-half of all sputum-scarce or smear-negative TB HIV-infected patients with a CD4 $\leqslant 200$ cells per $\mathrm{mL}$ who would have otherwise required further investigation.

Importantly, the LAM strip-test was particularly useful in patients with EPTB and those with a CD4 count $\leqslant 100$ cells per $\mathrm{mL}$, potentially offering a rapid rule-in diagnosis in these diagnostically challenging patient subgroups. Thus, a convenience urine sample could be obtained in patients with PTB and/ or EPTB irrespective of whether the patient could produce sputum. It is in this context, that the LAM assay may also offer advantage over other novel diagnostic formats, such as the Xpert MTB/RIF, as it obviates the need for a biological sample from the site of disease. Thus, although the assay is useful in only a specific patient subgroup, we believe that these results are encouraging given that the urine LAM strip-test is a simple-toperform bedside test that is affordable (less than US\$3.50 per test), rapid (providing results within $35 \mathrm{~min}$ ) and uses an easily obtainable urine sample. These factors make it ideally suited for use in hospitals in high-burden TB-HIV settings where resources and laboratory infrastructure are limited.

What is an optimal and pragmatic cut-off point to select when using the LAM strip-test? The manufacturer recommends the grade 1 cut-off point (fig. 1), and this cut-off point proved reliable and specific in a recently published study in an outpatient antiretroviral-screening clinic [19]. However, in our study, using the grade 1 cut-off point the LAM strip-test had suboptimal specificity (in both analyses) and modest interobserver agreement, thereby limiting rule-in test utility. Moreover, it was noted that, when the faintest bands were being interpreted in differing ambient light conditions, readings were variable. By contrast, using the grade 2 cut-off point was considerably more reliable and improved test specificity, and is hence the one that we recommend. The reasons for differing cut-off point-specific performance differences between the data of LAWN et al. [19] and our data remains unclear, but may be related to within batch variability, impact of storage duration and conditions, and reader blinding and experience. Further prospective standardised studies are now urgently required to clarify these findings and the optimal rule-in cut-off point in different settings.

Given the known limitations of TB culture as a reference standard, especially amongst HIV-infected hospitalised TB patients (very low sputum baciliary load and sputum scarce), we performed two analyses of diagnostic accuracy using two reference standards. Furthermore, a control group of HIV-infected non-TB patients was enrolled to obtain an additional estimate of test specificity. Specificity measures showed significant variation between different patient groups and analyses, with analysis 1 likely to provide an underestimate and analysis 2 an overestimate of test specificity. We feel that, given this particular patient population, and the large number of probable (likely) TB cases in the cohort (including smear-positive culture negative patients), analysis 2 test specificity is a more representative and accurate. Larger studies using a standardised TB diagnostic evaluation are now urgently required to clarify our findings.

Our study has a number of limitations. For practical reasons, our study did not use standardised TB evaluation, and diagnostic work-up was at the discretion of the attending clinician. This may have introduced work-up bias. Our study used frozen samples to 
evaluate the LAM strip-test and this may have impacted test performance. However, sample freeze-thaw showed no significant effect in our preliminary experiments or in recent metaanalysis of studies using the TB LAM ELISA [13]. The LAM strip-test does not inform on drug susceptibility and thus additional samples may be required, if appropriate, to make a diagnosis of multi-drug resistant (MDR)-TB. This consideration, hitherto of limited general applicability, may become more relevant in the future when National TB Programmes in Africa develop the capacity to diagnose and treat MDR-TB. Finally, LAM test results were not used for treatment decisions or to guide further diagnostic work-up and, consequently, the clinical impact on patient mortality, time to diagnosis, length of hospital stay and cost-effectiveness could not be evaluated.

The novel LAM strip-test, although restricted to HIV-infected persons with advanced immunosuppression, targets a vulnerable population with high mortality and healthcare-related costs. Given its low cost and ease of use, it has the potential to offer significant clinical utility when combined with smear microscopy in high-burden district hospitals with poor or no laboratory infrastructure. If used to guide the rapid initiation of anti-TB treatment, the impact of which is supported by a recent study in HIV-infected hospitalised patients [4], LAM strip-testing has the potential to decrease TB-related mortality, length of hospital stay and the overall cost of the TB diagnostic work-up in resource-poor healthcare systems. Suboptimal specificity may limit this potential clinical utility. Larger prospective studies of diagnostic accuracy, using a standardised TB diagnostic evaluation, are now urgently required to clarify test specificity in hospitalised patients with TB-HIV co-infection and confirm our recommendation to use the grade- 2 cut-off point for optimising rule-in value and inter-reader reliability.

\section{SUPPORT STATEMENT}

Although Alere supplied the ELISA and LAM strip-tests free of charge they had no role in the design and conduct of the study, analysis of the data or writing of the manuscript. J.G. Peter and R. van Zyl-Smit are supported by the Fogarty International Clinical Research Scholars/ Fellows Support Centre National Institutes of Health grant R24TW007988, SATBAT and the EDCTP. G. Theron is supported by the EDCTP and NRF. K. Dheeda is supported by the EDCTP (TB NEAT and TESA) and the SA DST and NRF (SARChI).

\section{STATEMENT OF INTEREST}

A statement of interest for the study can be found at www.erj.ersjournals. $\mathrm{com} / \mathrm{site} / \mathrm{misc} /$ statements.xhtml

\section{ACKNOWLEDGEMENTS}

We thank the research nursing staff, including N. Kelly and F. Embrahim (University of Cape Town, Cape Town, South Africa), who were involved in the recruitment of patients and collection of urine samples. The support of the clinical and nursing staff of the general medical wards of Groote Schuur, Victoria, G.F. Jooste and New Somerset Hospitals (all Cape Town, South Africa) was greatly appreciated.

\section{REFERENCES}

1 WHO. Global tuberculosis control 2010. WHO/HTM/TB/2010.7. Geneva, World Health Organisation, 2010.

2 Getahun H, Gunneberg C, Granich R, et al. HIV infectionassociated tuberculosis: the epidemiology and the response. Clin Infect Dis 2010; 50: Suppl. 3, S201-S207.
3 Harrison D. An overview of health and health care in South Africa 1994-2010: priorities, progress and prospects for new gains; 2009. Online report.

4 Holtz TH, Kabera G, Mthiyane T, et al. Use of a WHO-recommended algorithm to reduce mortality in seriously ill patients with HIV infection and smear-negative pulmonary tuberculosis in south africa: an observational cohort study. Lancet Infect Dis 2011; 11: 533-540.

5 Cohen T, Murray M, Wallengren K, et al. The prevalence and drug sensitivity of tuberculosis among patients dying in hospital in kwazulunatal, South Africa: a postmortem study. PLoS Med 2010; 7: e1000296.

6 Cain KP, McCarthy KD, Heilig CM, et al. An algorithm for tuberculosis screening and diagnosis in people with HIV. N Engl J Med 2010; 362: 707-716.

7 Monkongdee P, McCarthy KD, Cain KP, et al. Yield of acid-fast smear and mycobacterial culture for tuberculosis diagnosis in people with human immunodeficiency virus. Am J Respir Crit Care Med 2009; 180: 903-908.

8 Van Rie A, Page-Shipp L, Scott L, et al. Xpert( $(\mathbb{R})$ MTB/RIF for pointof-care diagnosis of TB in high-HIV burden, resource-limited countries: hype or hope? Expert Rev Mol Diagn 2010; 10: 937-946.

9 Mishra AK, Driessen NN, Appelmelk BJ, et al. Lipoarabinomannan and related glycoconjugates: structure, biogenesis and role in Mycobacterium tuberculosis physiology and host-pathogen interaction. FEMS Microbiol Rev 2011; 35: 1126-1157.

10 Hamasur B, Bruchfeld J, Haile M, et al. Rapid diagnosis of tuberculosis by detection of mycobacterial lipoarabinomannan in urine. J Microbiol Methods 2001; 45: 41-52.

11 Tessema TA, Bjune G, Hamasur B, et al. Circulating antibodies to lipoarabinomannan in relation to sputum microscopy, clinical features and urinary anti-lipoarabinomannan detection in pulmonary tuberculosis. Scand J Infect Dis 2002; 34: 97-103.

12 Peter J, Green C, Hoelscher M, et al. Urine for the diagnosis of tuberculosis: current approaches, clinical applicability, and new developments. Curr Opin Pulm Med 2010; 16: 262-270.

13 Minion J, Leung E, Talbot E, et al. Diagnosing tuberculosis with urine lipoarabinomannan: systematic review and meta-analysis. Eur Respir J 2011; 38: 1398-1405.

14 Gounder CR, Kufa T, Wada NI, et al. Diagnostic accuracy of a urine lipoarabinomannan enzyme-linked immunosorbent assay for screening ambulatory HIV-infected persons for tuberculosis. J Acquir Immune Defic Syndr 2011; 58: 219-223.

15 Flores L, Steingart K, Dendukuri N, et al. Systematic review and meta-analysis of antigen detection tests for the diagnosis of tuberculosis. Clin Vaccine Immunol 2011; 18: 1616-1627.

16 Shah M, Variava E, Holmes CB, et al. Diagnostic accuracy of a urine lipoarabinomannan test for tuberculosis in hospitalized patients in a high HIV prevalence setting. J Acquir Immune Defic Syndr 2009; 52: 145-151.

17 Lawn SD, Edwards DJ, Kranzer K, et al. Urine lipoarabinomannan assay for tuberculosis screening before antiretroviral therapy diagnostic yield and association with immune reconstitution disease. AIDS 2009; 23: 1875-1880.

18 Alere. Alere Determine TB LAM Ag rapid test product listing. 2011. www.alerehiv.com/hiv-comorbidities/features

19 Lawn SD, Kerkhoff AD, Vogt M, et al. Diagnostic accuracy of a low-cost, urine antigen, point-of-care screening assay for HIVassociated pulmonary tuberculosis before antiretroviral therapy: a descriptive study. Lancet Infect Dis 2012; 12: 201-209.

20 Dheda K, Davids V, Lenders L, et al. Clinical utility of a commercial LAM-ELISA assay for TB diagnosis in HIV-infected patients using urine and sputum samples. PLoS One 2010; 5: e9848.

21 Landis JR, Koch GG. The measurement of observer agreement for categorical data. Biometrics 1977; 33: 159-174.

22 Bossuyt PM, Reitsma JB, Bruns DE, et al. The STARD statement for reporting studies of diagnostic accuracy: explanation and elaboration. The standards for reporting of diagnostic accuracy group. Croat Med J 2003; 44: 639-650. 